\author{
P Anjaneyulu, B Annapurna, P Haribabu, T Vijetha \& R Karthik \\ Department of Electronics and Communication Engineering \\ MLR Institute of Technology, Hyderabad-500043, India
}

Abstract: This paper presents a mini review on the basics of Radars. The parameters considered for determining the target location are explained in detail. Also the concept of Jamming and various types are explained in detail.

Keywords: Antenna, Radar, Jamming, Communication

\section{INTRODUCTION}

RADAR is an object detection system that uses electromagnetic waves to identify the range, altitude, direction, or speed of both moving and fixed objects such as aircraft, ships, motor vehicles, weather formations, and terrain [1].

A Radar system has a transmitter that emits microwaves or radio waves. These are reflected by the target back to a receiver, usually in the same location as the transmitter. Although the signal returned is usually very weak, the signal can be amplified and displayed. This enables Radar to detect objects at ranges where other emissions, such as sound or visible light, would be too weak to detect. Radar is used in meteorological detection of precipitation, measuring ocean surface waves, air traffic control, police detection of speeding traffic, and by the military.

RADAR basically consists of transmitter, receiver and antenna. The transmitter generates an electromagnetic signal, which is radiated into space by an antenna [2]. The antenna is usually shared on a time-shared basis. A portion of the transmitted energy is blocked by the target and reradiated or reflected in many directions. This is collected by the Radar antenna, which delivers it to the receiver. The receiver then processes it to detect the presence of the target and to determine its location. The principle of Radar is as shown in figure 1.

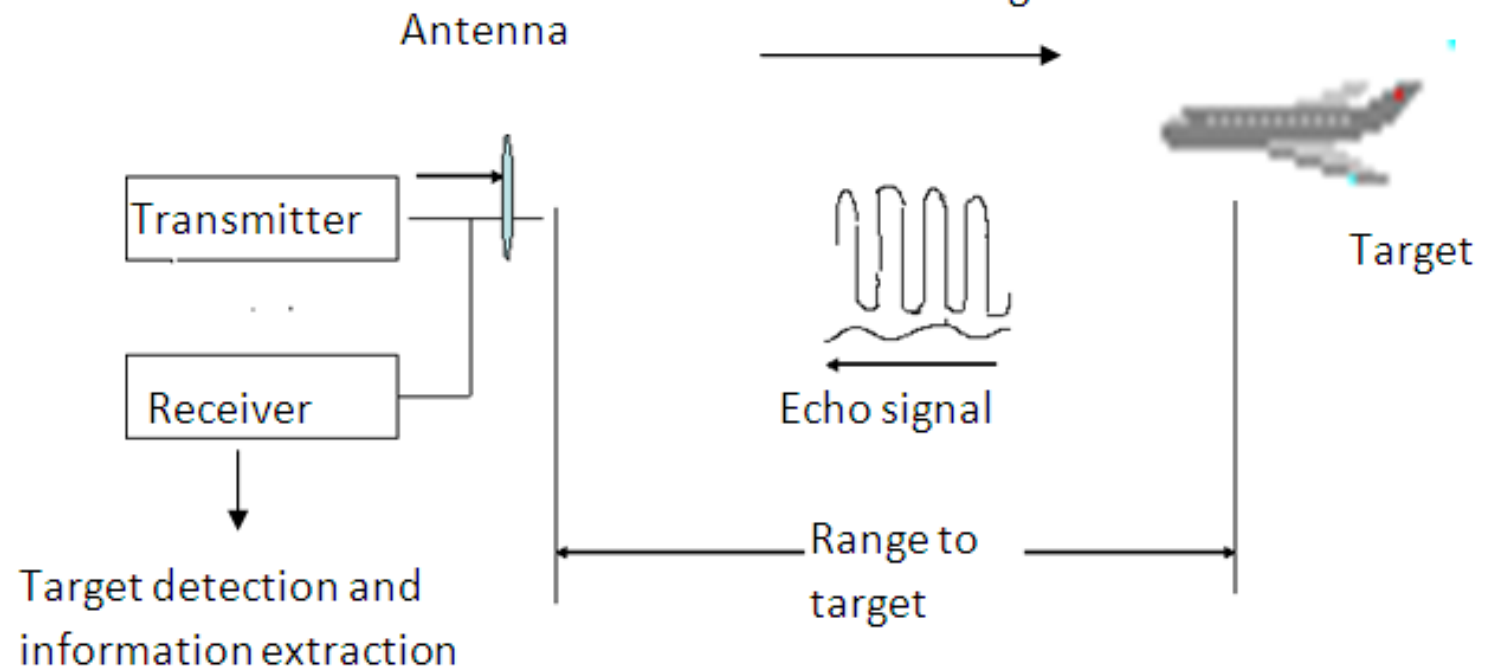

\title{
Transmitted signal
}

Figure 1: RADAR Principle

The electronics principle on which Radar operates is very similar to the principle of sound-wave reflection. If you shout in the direction of a sound-reflecting object (like a rocky canyon or cave), you will hear an echo. If you know the speed of sound in air, you can then estimate the distance and general direction of the object. The time required for a return echo can be roughly converted to distance if the speed of sound is known. Radar uses electromagnetic energy Radar equation is as follows [1]: pulses in much the same way, as shown in figure 1 . The radio-frequency (RF) energy is transmitted to and reflects from the reflecting object. A small portion of the energy is reflected and returns to the Radar set. This returned energy is called an ECHO, just as it is in sound terminology. Radar sets use the echo to determine the direction and distance of the reflecting object. 
Received Power $=$ Transmitted Power X Fraction of transmitted power that is reflected To receiver $X$ Fraction of reflected power that is intercepted by Receiving antenna

$P_{r}=\frac{P_{t} g_{t} g_{r} \lambda^{2} \sigma_{B}}{(4 \pi)^{3} R_{t}^{2} R_{r}^{2}}$

For determining the target location three pieces of information are required [2]:

1. Azimuth Angle

2. Elevation angle

3. Distance to target.

\section{Azimuth Angle:}

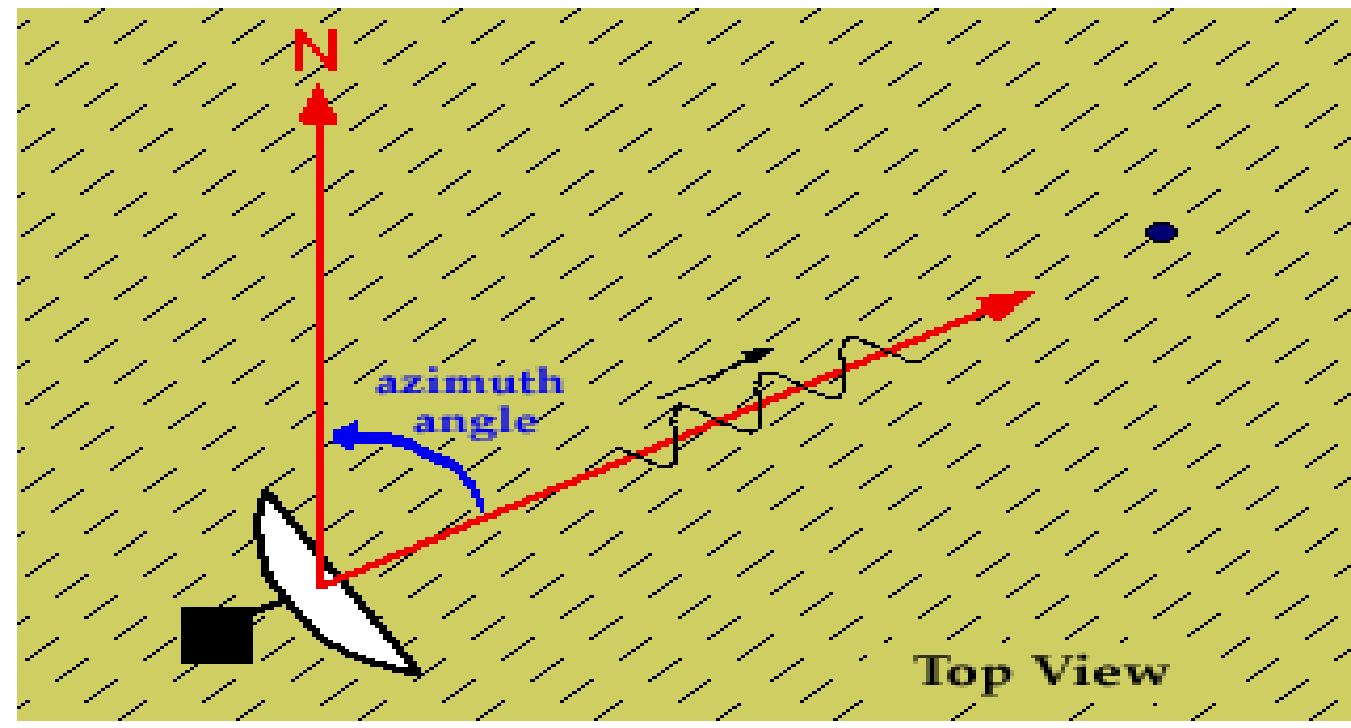

Figure 2: Azimuth Angle

The azimuth angle is nothing but angle of 'beam' with respect to the North.

\section{Elevation angle}

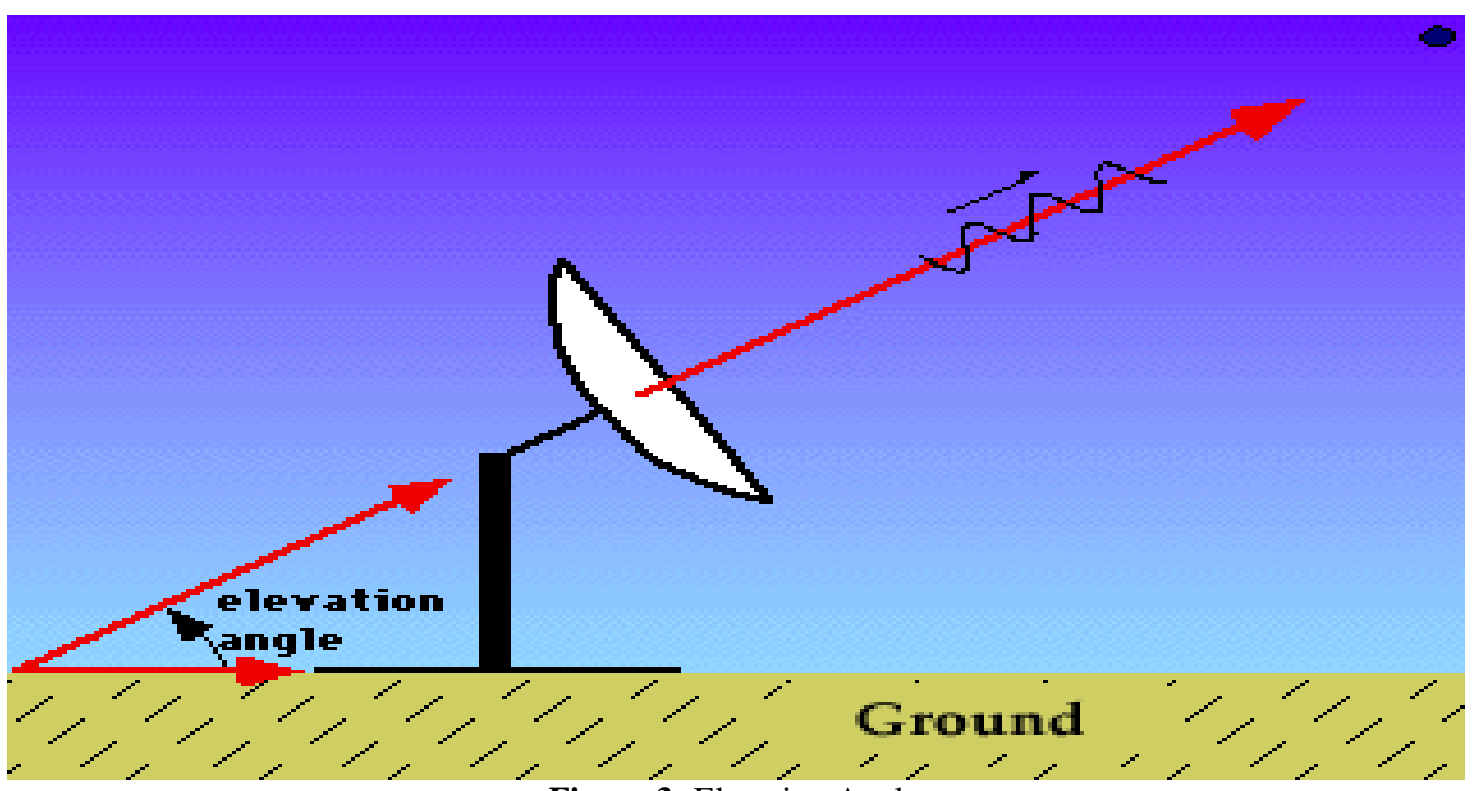

Figure 3: Elevation Angle

Elevation is angle is nothing but the angle of 'beam 'with respect to the ground. 


\section{Distance of Target}

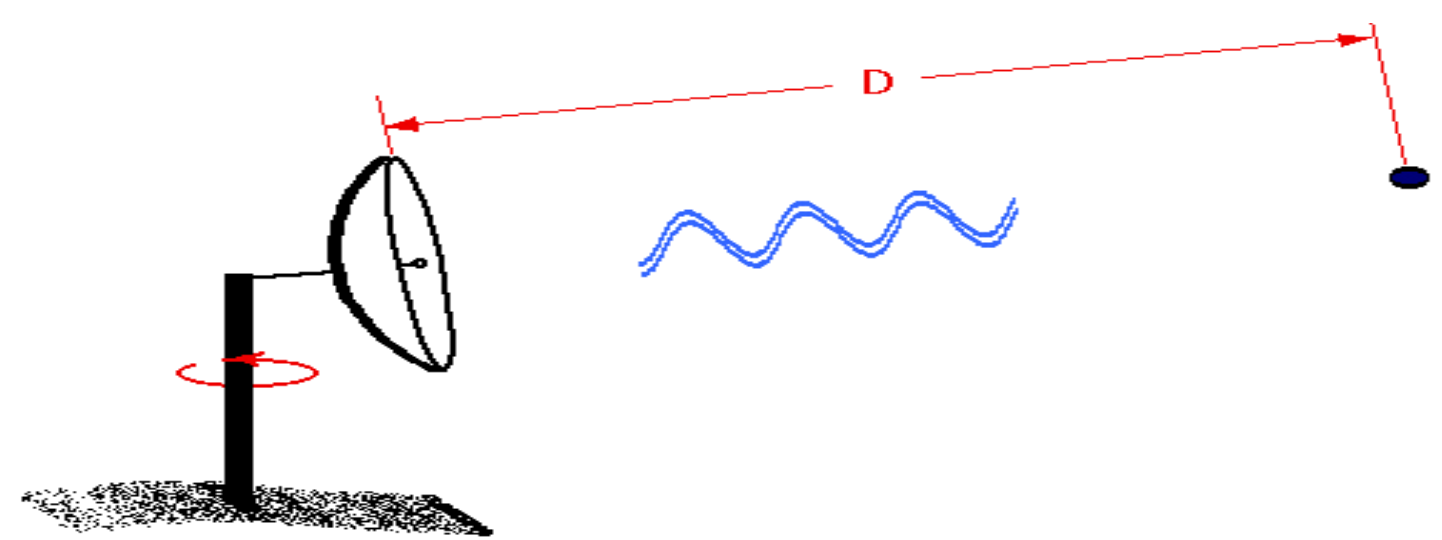

Figure 4: Distance of Target

The distance ' $\mathrm{D}$ ' of the target is given by:

$D=c T / 2$

Where; $\mathrm{T}=$ pulse's round trip time

$$
\mathrm{C}=\text { the velocity of light }
$$

\section{JAMMING IN RADAR SYSTEMS}

\section{PHYSICAL CONCEPT}

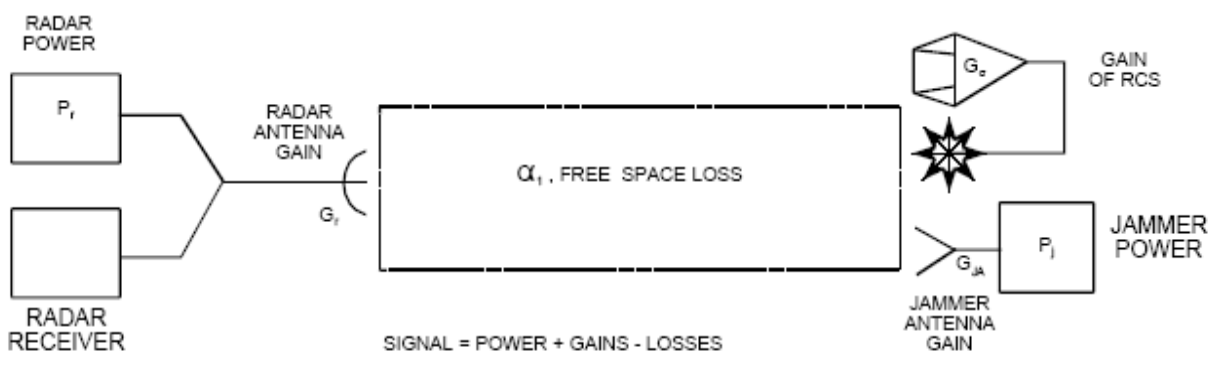

Figure 5: RADAR Jamming Visualised

Electronic Counter Measures (ECM) is that division of electronic warfare involving actions taken to prevent or reduce an enemy's effective use of the electromagnetic Spectrum [3, 4]. Radar Jamming is an Electronic Counter Measure (ECM) technique to prevent, delay, or confuse the Radar processing of target information. There are many methods and techniques to jam Radar receiver.

Jamming can be classified as based on presence of internal radiation source as:

- Active jamming

- Passive jamming

Jamming also can be classified based on property of jamming function as:

- $\quad$ Suppression jamming

- Deception jamming

Deception jammer senses the incoming radar signals and replicates the simulated target echoes in order to confuse radars, hindering the ability to identify true targets from false targets [5].

Basically, there are two different methods of employing active ECM against hostile radars:

- Self Protection ECM

- $\quad$ Support ECM

Jamming is part of Electronic Warfare [6]. It is an Electronic Counter-Measure, and thus its purpose is to deny the enemy the ability to make use of the electromagnetic spectrum. Jamming works by filling the receiver of the enemy's equipment with "noise." Generally the target of a jammer is the enemy's radar systems. 


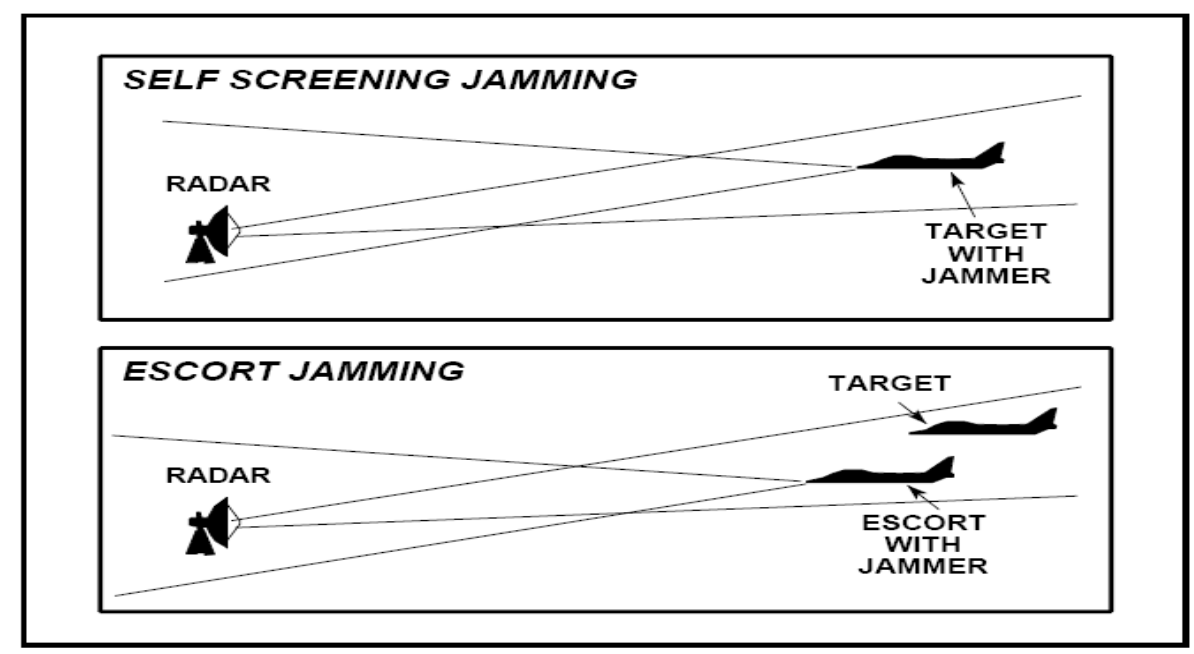

Fig.6: Self Protections and Escort Jamming [7]

\section{SELF PROTECTION ECM}

Self Protection ECM is often called "self screening jamming" and also "DECM", which is an acronym for either "Defensive ECM" or "Deception ECM". Deception uses ECM to forge false target signals that the radar receiver accepts and processes as real targets.

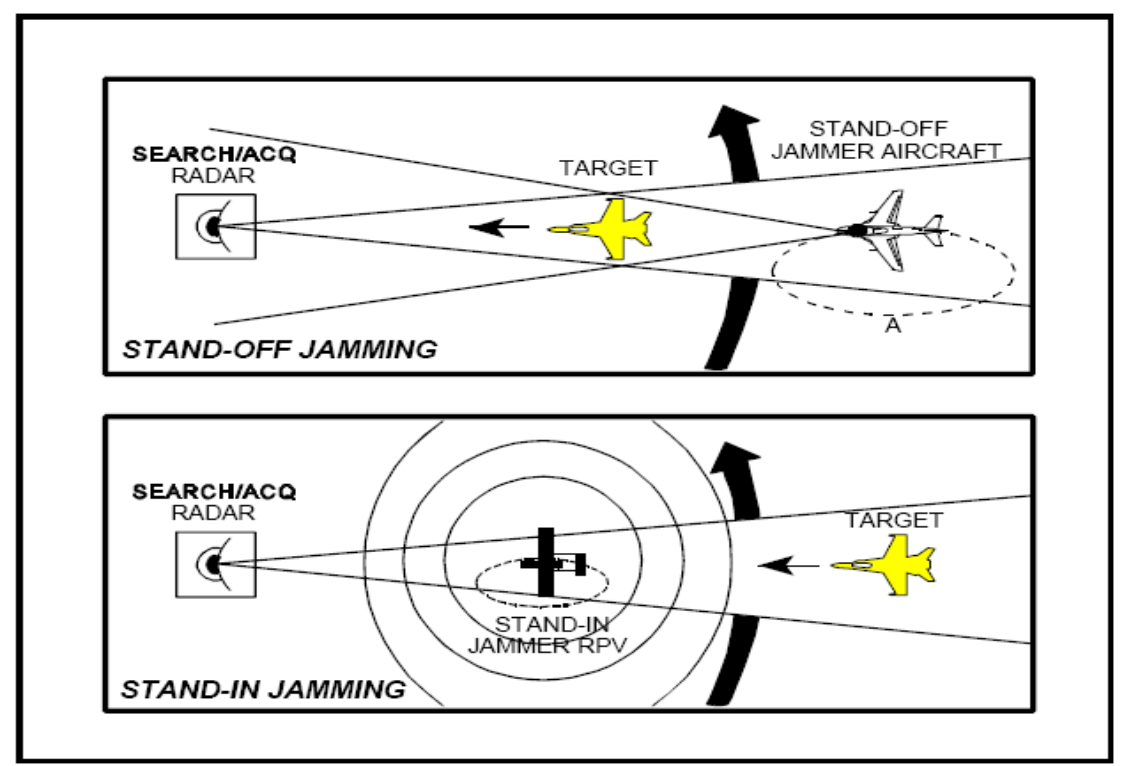

Fig.7: SIJ \& SOJ

\subsection{Active Suppression Jamming}

Active suppression jamming can beclassified as modulation wave and non-modulation wave jamming [6, 7]. Nonmodulation wave jamming is used rarely as its effect is poor. Modulation wave jamming can be classified as regular signal modulation and noise modulation. Regular signal modulation includes sine, pulse, and sawtooth modulation. Noise modulation includes noise amplitude modulation, noise frequency modulation and synthetic modulation jamming of noise amplitude and frequency. They are widely used for they can generate large power and wideband jamming signal.

\subsection{Active Deception Jamming}

Generally active deception jamming simulates target echo using a jamming pulse to deceive the Radar system and operator. Thus, making it difficult to distinguish between true and false targets or interpret a false target as a true target [6]. Generally there are angle deception, range deception, and velocity deception jamming against tracking Radar. Against warning and guidance Radar, however, there is false target jamming.

\subsection{Passive Jamming}

This technique uses special made material (Radar cross section reduction (RAM)) to reflect electromagnetic waves or change the travel condition to jam Radar [6].

\section{CONCLUSION}

This paper presents a mini review on the basics of Radars. The parameters considered for determining the target location are explained in detail. Also the concept of Jamming and various types are explained in detail. 


\section{REFERENCES}

1. I. Merrill Skolnik, "Introduction to RADAR systems", Tata McGraw-Hill publishing company limited. Singapore, $3^{\text {rd }}$ edition 2001.

2. A.K Subramanian, "Jamming primer", Defense Scientific Information \& Documentation Center Defense Research \& Development Organization Ministry of Defense , India, $2^{\text {nd }}$ edition, 2003.

3. A. K Subramanian, "Modeling Radar-ECCM A System Approach", Defense Scientific Information \& Documentation
Center Defense Research \& Development Organization Ministry of Defense, India, $2^{\text {nd }}$ edition 2003.

4. L.Stephen Johnston, "Radar Electronic CounterCountermeasures", IEEE Transaction on Aerospace and Electronic Systems, Vol-14, pp.1-8, 1978.

5. "A Survey of Radar ECM and ECCM", IEEE Transaction on Aerospace and Electronic Systems, Vol 31, No.3, pp. 1-6, 1995.

6. D.John Mosinski, "Electronic Countermeasures", IEEE Transaction on Aerospace and Electronic System, Vol 13, No.2, pp. 1-6, 2002. 